\title{
The epidemiology of patellofemoral disorders in adulthood: a review of routine general practice morbidity recording
}

\author{
Laurence Wood, Sara Muller and George Peat \\ Arthritis Research UK Primary Care Centre, Keele University, Keele, Staffordshire, UK
}

\begin{abstract}
Aim: To describe the annual consultation prevalence of different patellofemoral disorders across the adult life span. Background: The knee is the second most common site for musculoskeletal pain. Evidence from sports injury and orthopaedic settings suggests that patellofemoral disorders constitute a significant proportion of knee pain cases, but we have no evidence from general practice - the setting where most patellofemoral problems will present and be dealt with - of the prevalence of consultations for these problems in the different age groups. Methods: Age- and gender-stratified annual person consulting prevalence rates (APCPRs) for patellofemoral disorders were calculated using 2006 data from the 'Consultations in Primary Care Archive' (CiPCA) - a fully audited database of continuous morbidity recording used by the general practitioners (GPs) in eight general practices in North Staffordshire. Findings: A total of $57555>15-$ year-olds were registered with the eight general practices in 2006; 1782 made a kneerelated consultation, of which 303 (one-in-six) were coded as patellofemoral disorders (APCPR, 53 per 10000 persons), suggesting that GPs consider a significant proportion of adult knee pain consulters to have patellofemoral disorders. Twelve patellofemoral disorders diagnosed by the GPs were identified. The non-specific diagnosis of 'anterior knee pain' (AKP) was by far the most common (APCPR, 37.2/10000). Other more specific diagnoses were rare by contrast, suggesting that specific patellofemoral disorders are rarely diagnosed in general practice (consultation prevalences, in order of most prevalent first: bursitis (7.8/10 000), patellofemoral osteoarthritis (2.3/10 000); APCPRs for the remaining nine diagnoses ranged between 0 and 1.6/10000). However, the use of alternative less anatomically specific diagnostic codes, such as for 'knee pain', may mean that our estimate of one-in-six is an underestimate of GPs' true attribution rates to patellofemoral joint disorders of people consulting them with knee pain. AKP was seen equally commonly by GPs across all age strata, contradicting the received wisdom that AKP problems are most common among younger adults.
\end{abstract}

Key words: anterior knee pain; consultation prevalence; epidemiology; general practice; patellofemoral disorders; primary care

Received 4 June 2010; accepted 1 November 2010; first published online 1 February 2011

\section{Introduction}

After low back pain, the knee is the second most common site for musculoskeletal pain in

Correspondence to: Dr Laurence Wood, Arthritis Research UK Primary Care Centre, Keele University, Staffordshire ST5 5BG, UK. Email: 1.r.j.wood@cphc.keele.ac.uk

(C) Cambridge University Press 2011 adulthood in the United Kingdom (Urwin et al., 1998; McAlindon, 1999; Peat et al., 2001). Patellofemoral joint (PFJ) disorders, presenting as anterior knee pain (AKP), are recognised to be a major component of knee pain cases seen in sports injury and orthopaedic outpatient clinics (Devereaux and Lachmann, 1984; Stanitski, 1993; Luhmann et al., 2008), and specialists from these fields consider 
there to be many different treatments that are specific to the particular causes of patellofemoral pain (Juhn, 1999; Fulkerson et al., 2004; Post, 2005; Witvrouw et al., 2005; Fagan and Delahunt, 2008). Yet, there is an almost total absence of evidence on whether PFJ disorders are diagnosed and treated in general practice, despite the fact that knee pain problems are one of the most common reasons for people consulting their GPs (van der Waal et al., 2006; Royal College of General Practitioners' Birmingham Research Unit, 2007). This study aimed to explore the extent to which GPs make PFJ-specific diagnoses, what these diagnoses are, and whether they are more commonly made in certain genders and age groups than in others.

\section{Methods}

Annual age-stratified person consulting prevalence rates for patellofemoral disorders were calculated using 2006 data from the 'Consultations in Primary Care Archive' (CiPCA) - a fully audited computerised database of continuous morbidity recording used by the general practitioners (GPs) in eight general practices in North Staffordshire (Jordan et al., 2007) (ethical approval for CiPCA was granted by the North Staffordshire Research Ethics Committee [LREC Ref. No.03/04]). This was done in the following way. First, we identified all musculoskeletal knee-related Read codes, using the list generated by Jordan et al. (2010). Second, we excluded all non-diagnostic codes (ie, those that were used to indicate a clinical process, such as the ordering of X-rays or referral to secondary care). Third, we selected all those codes that related specifically to patellofemoral disorders and categorised these on the basis of presumed underlying pathophysiology (Table 1).

Fourth, we discounted repeated consultations by individuals given Read codes for the same patellofemoral disorders, in order to give an annual persons consulting prevalence rate. Fifth, we calculated the rate of consultation per 10000 population for each diagnostic category. Finally, in order to determine whether some of these diagnostic Read codes are used more commonly for one gender than the other or in some age groups than in others, we stratified these consultation rates both by gender and by five 15 -year age bands from the age of 15 years. To contextualise
Table 1 Proposed categorisation of Read-coded consultations for patellofemoral disorders

\begin{tabular}{lc}
\hline Patellofemoral disorders & $\begin{array}{l}\text { Number of } \\
\text { corresponding } \\
\text { Read codes }\end{array}$ \\
\hline Patellofemoral osteoarthritis & 1 \\
Anterior knee pain & 2 \\
Osteochondritis & 3 \\
Patellar maltracking/subluxation/ & 22 \\
$\quad$ dislocation & 9 \\
Other patella & 2 \\
Patellofemoral disorder & 2 \\
Traction enthesopathies & 1 \\
Chondromalacia & 20 \\
Fractures/fracture dislocations & \\
$\quad$ of the patella & 3 \\
Patellar tendonitis & 10 \\
Pre- or infra-patellar bursitis & 2 \\
Patellar tendon rupture &
\end{tabular}

Read codes/terms are available as an online appendix.

our results, we calculated prevalence estimates for consultations coded as knee osteoarthritis (OA; based on eight Read codes and excluding those specifically labelled as patellofemoral OA) for comparison, since $\mathrm{OA}$ is the most common joint disease (The National Collaborating Centre for Chronic Conditions) and a common cause of GP consultation (McCormick et al., 1995). In order to assist in interpretation of the results and due to the variable numbers of people consulting between categories, we calculated 95\% CI (using the -cicommand with the binomial option in Stata 9.2).

\section{Results}

Of the 57555 individuals aged $\geq 15$ years registered with the eight general practices in 2006, 1782 made a knee-related consultation, of which 303 $(17 \%)$ were coded as patellofemoral disorders, giving an annual persons consulting prevalence rate for PFJ disorders of 53 per 10000 persons. Ageand gender-stratified person consultation rates for all PFJ disorders are given in Table 2. Generally, the prevalence rates (expressed as a proportion of persons registered with the GP practices in each age and gender stratum) did not differ greatly between the different strata. Two possible exceptions to this are worthy of note: in both the 30-44 and the $\geq 75$ years age groups, there appear to be 
Table 2 Number of persons registered with the general practices and persons' consultation prevalence rates by age and gender strata

\begin{tabular}{lllll}
\hline Age band (years) & Gender & $\begin{array}{l}\text { Number of } \\
\text { persons } \\
\text { registered }\end{array}$ & $\begin{array}{l}\text { Number of } \\
\text { persons consulting } \\
\text { with PFJ disorder }\end{array}$ & $\begin{array}{l}\text { Persons consulting } \\
\text { prevalence rate } \\
\text { (per 10 000) }\end{array}$ \\
\hline Total sample ( $\geq 15)$ & Female and male & 57555 & 303 & 52.6 \\
& Female only & 29611 & 154 & 52.0 \\
$15-29$ & Male only & 27944 & 149 & 53.3 \\
& Female and male & 12248 & 73 & 59.6 \\
& Female only & 6062 & 37 & 61.0 \\
$30-44$ & Male only & 6186 & 36 & 42.5 \\
& Female and male & 14588 & 62 & 37.5 \\
$45-59$ & Female only & 7209 & 27 & 47.4 \\
& Male only & 7379 & 35 & 56.1 \\
$60-74$ & Female and male & 14270 & 80 & 57.0 \\
& Female only & 7198 & 41 & 55.1 \\
& Male only & 7072 & 39 & 57.6 \\
$\geq 75$ & Female and male & 10598 & 61 & 55.9 \\
& Female only & 5545 & 31 & 59.4 \\
& Male only & 5053 & 30 & 46.1 \\
& Female and male & 5851 & 27 & 50.0 \\
& Female only & 3597 & 18 & 39.9 \\
\hline
\end{tabular}

PFJ = Patellofemoral joint.

fewer person consultations. In the 30-44 years age group, this seems to be particularly the case among female consulters, whereas, in the $\geq 75$ age group, it is the male consulters who seem to be especially infrequent.

Categories with no individuals consulting in three out of the five age strata were discounted from further analyses. These were 'osteochondritis' (zero persons consulting in the study period), 'maltracking/subluxation/dislocation' $(n=7)$, 'other patella' $(n=1)$, 'patellofemoral disorder' $(n=1)$, 'traction enthesopathies' $(n=9)$, 'fractures/ fracture dislocations' $(n=5)$ and 'patellar tendon rupture' $(n=1)$. Age-stratified persons consulting prevalence rates (per 10000 individuals) for the remaining patellofemoral disorders are given in Figure 1, together with the comparator, knee OA. In view of similar prevalence rates across the genders and the relatively small numbers within each age-sex stratum, we present the prevalence rates for males and females combined.

'AKP' was by far the most common category of patellofemoral disorders recorded by GPs (persons consulting prevalence rate per $10000=37.2$ ), accounting for $12 \%$ of all knee-related consulters and $71 \%$ of all persons consulting and diagnosed with a patellofemoral disorder. Contrary to expectations, the consultation prevalence rate of AKP was relatively constant across the entire adult age range. 'Bursitis' was the next most common (persons consulting prevalence rate per $10000=7.8$ ), with prevalence rates again broadly similar across all ages. Cases of GP-diagnosed chondromalacia and patella tendonitis were rare and appeared mainly in adolescents and younger adults. In contrast to knee OA, there were few cases specifically diagnosed as PFJ OA $(n=13)$, with the majority occurring in adults aged $\geq 60$ years.

\section{Discussion}

We estimate that one in six adults in the UK consulting general practice for a knee problem will be clinically suspected or diagnosed as having a patellofemoral disorder. Generally speaking, the overall persons consulting prevalence rate of patellofemoral disorders appears to be fairly consistent across the age and gender strata. The possible exceptions to this are the 30-44 years age group, where fewer women, in particular, were ascribed consultation codes for patellofemoral disorders, and the $\geq 75$ years age group, where markedly fewer 


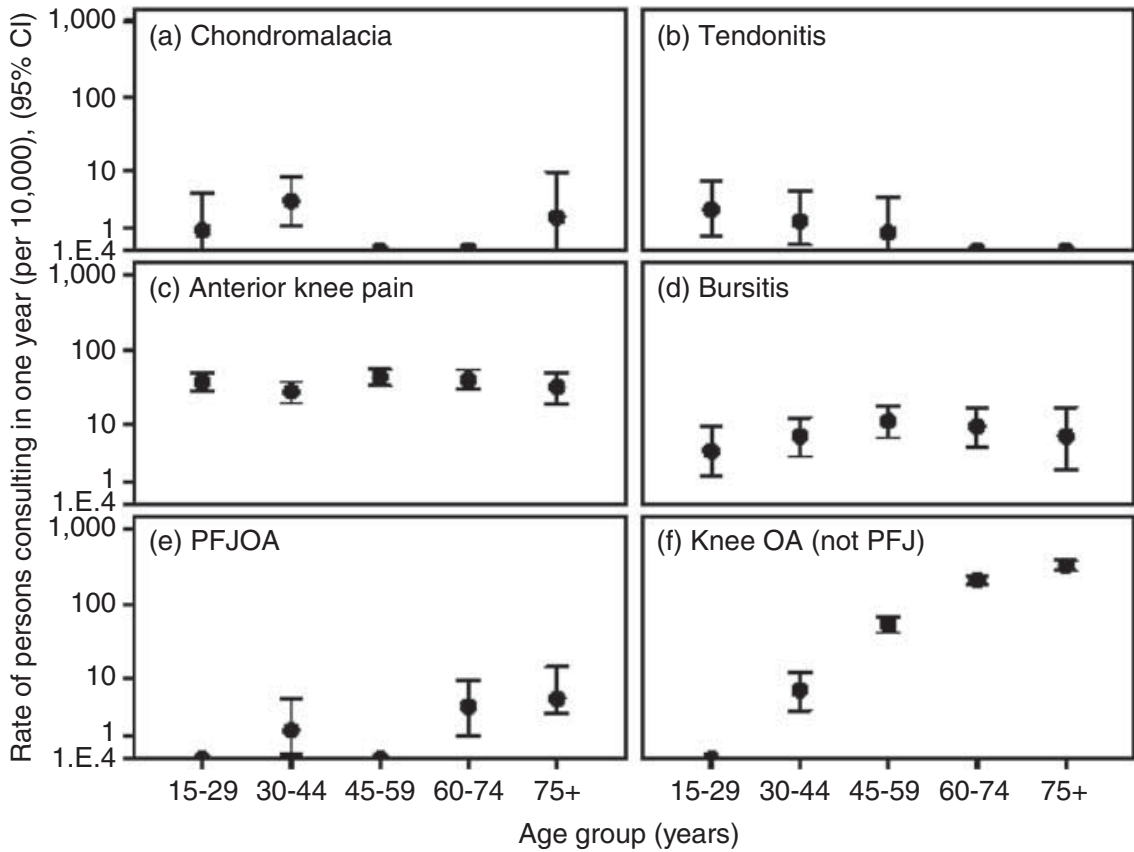

Figure 1 Annual persons consulting prevalence rates for patellofemoral disorders by age band, with knee osteoarthritis (not patellofemoral) for comparison ( $y$ axis is logarithmic scale)

men were ascribed these consultation codes. Before jumping to hypothesise what might be the cause of these exceptions, it is worthwhile noting that the numbers involved here are so small, particularly in the case of the $\geq 75$ years age group, that these variations may be random in nature.

Although the symptom code of 'AKP' is fairly commonly used by GPs across the age range of adolescents and adults consulting with knee problems, diagnoses of the specific patellofemoral disorders that may underlie such pain are not. While the term 'AKP' is clearly preferable in most circumstances to the historically oft-misused and misleading diagnosis of chondromalacia patellae (Fulkerson et al., 2004; Grelsamer, 2005; Pihlajamaki et al., 2010), it has to be borne in mind that it may cover a whole raft of pathological causes (one authority lists 26 possibilities [Macnicol, 1995]) that may require very different treatment approaches, both conservative and surgical.

As previously stated, there is a striking absence of evidence in the literature on the basic epidemiology of most patellofemoral disorders. AKP was found in this study to be the most commonly used 'diagnostic' code. One study from the United States attempted to quantify referrals for AKP from physicians to a primary care sports medicine clinic associated with a large managed care system (Butcher et al., 1996). The majority of these referrals were from family practice clinic providers, which included physicians, physician assistants and nurse practitioners. This study reported that out of 1857 patient contacts, $10.6 \%$ were for chronic AKP (Butcher et al., 1996). While these data can hint at the scale of the problem, they cannot give a true picture of consultation prevalence at the primary care level. Van Middelkoop et al. (2008) selected active sportspeople and non-athletes from their cohort of knee pain consulters in primary care, which was spread across 40 different GPs in the south-western Netherlands (van Middelkoop et al., 2008). They found that $11.0 \%$ of these individuals were given a working diagnosis of 'patellofemoral pain syndrome' by their GP, compared with $12.0 \%$ given a Read Code for 'AKP' in the current analysis.

Published data regarding the prevalence of the other less-commonly used non-OA patellofemoral disorders in people with knee pain are 
similarly sparse. In their study of knee pain and radiographic OA in participants drawn from Veterans Affairs medical centres and the community, Hill et al. (2003) found that prepatellar and superficial infrapatellar bursitis were present in $12 \%$ of participants with knee pain and radiographic OA. One Chinese study of the comparative prevalence rates of chondromalacia among college students from a gymnastics department and students from a non-gymnastic department found the prevalence of chondromalacia patellae among the non-gymnasts to be $6 \%$ (although it should be made clear that their definition of chondromalacia patellae was akin to what is more commonly described as 'AKP' or 'patellofemoral pain syndrome', the diagnosis being arrived at on the basis of a clinical examination by a surgeon; Zhang et al., 2003). The use of Read codes for these diagnoses was found to be uncommon in our review of consultation data: only $2.5 \%$ of per person kneerelated consultations in 2006 were given one of the diagnostic codes pertaining to bursitis; the percentage for chondromalacia patellae was 0.4 .

Patellofemoral OA, as a clinical entity, has attracted attention in recent years (Arendt, 2005; Donell and Glasgow, 2007; Hinman and Crossley, 2007; Kalichman et al., 2007; Becker et al., 2008; Crossley et al., 2009; Duncan et al., 2009; Neuman et al., 2009). Cross-sectional community-based research studies suggest that structural changes consistent with OA may be more common in the PFJ than in the tibiofemoral joint (Lanyon et al., 1998; Duncan et al., 2006; Szebenyi et al., 2006), and are likely to be an important source of symptoms in knee OA (Hunter et al., 2003; Englund and Lohmander, 2005; Kornaat et al., 2006; Duncan et al., 2009). Indeed, one of these showed that approximately a quarter of $>50$-year-olds who had experienced some knee pain in the past year had isolated patellofemoral OA in at least one of their knees (Duncan et al., 2006). Therefore, it may seem strange that, while the annual incidence of new consultations for symptomatic knee OA has been estimated to be $0.5 \%$ (or 50 per 10, 000 individuals) of adults $>55$ years in the general population (Peat et al., 2001), the annual per person consultation rate for patellofemoral OA in the same age group should be as low as seven per 10000 , according to our review of general practice consultation data. This disparity may well be a reflection of the differential use of alternative diagnostic codes for a presenting problem by different GPs. Alternative codes often differ in the extent to which they are symptom-descriptive or patho-anatomic; hence, for instance, a diagnostic label of 'knee pain', 'OA of the knee' or 'patellofemoral OA' variously may be given for the older adult consulting with the same knee problem. Whether a GP uses one code or another is likely to vary from GP to GP (Jordan et al., 2006) and may be both a reflection of the extent to which they are confident in or comfortable with the diagnosis and also, at least in part, a matter of habit (Jordan et al., 2006).

This study used data from the CiPCA. GPs involved in this project undergo regular cycles of training, assessment and feedback in the quality of their computerised morbidity coding (Porcheret et al., 2004; Jordan et al., 2004; Jordan et al., 2007). Over $90 \%$ of doctor contacts in these practices are given a morbidity code (Porcheret et al., 2004). Such routine general practice consultation data constitute an important epidemiological resources that have the capacity to tell us much about consultation behaviour. However, it clearly has limitations when seeking information on the occurrence of specific patellofemoral disorders across the life course due to the fact that it captures only those complaints presented to, recognised and coded by the GP.

Use of only one year of consultation data resulted in small numbers of observations, which were insufficient for quantitative analysis. While expanding the period of consultation (eg, to five years) would enable more precise estimates to be made, and for the age-related pattern to be more clearly discerned, the overall conclusion that the diagnosis of specific patellofemoral disorders is rare in general practice - would not be expected to change.

Our findings imply that 'de novo' population studies are needed to describe the occurrence of patellofemoral disorders in the general population. The implications for clinical practice are less clear. It appears that specific patellofemoral disorders are not being diagnosed in general practice. But does this matter? In an article about the taxonomical confusion of terminologies for patellofemoral conditions, Grelsamer (2005) suggests that basket diagnoses, such as 'AKP', should be abandoned in favour of diagnoses that are 
more specific to the source of a patient's pain. To our mind, the answer to the question 'Does this matter?' depends on three factors: 1) The GPs' capacity to diagnose specific patellofemoral disorders without the aid of sophisticated imaging techniques or arthroscopic examination. Authorities suggest that it is the clinical history taking and physical examination, rather than such techniques, that is central to the accurate diagnosis of patellofemoral disorders (LaBella, 2004; Brukner et al., 2007). 2) The extent to which these disorders need treating because they will not get better without intervention. Recent evidence, contrary to the traditional view of AKP problems being essentially benign and self-limiting (Sandow and Goodfellow, 1985), suggests that symptoms may persist for many years into adulthood (Stathopulu and Baildam, 2003) and may even pre-date the development of patellofemoral OA in later life (Utting et al., 2005). 3) The existence of effective treatments specific to the particular patellofemoral diagnoses. Experts in the field are clear that there are many different treatments, conservative and otherwise, that are specific to the particular causes of patellofemoral pain (Juhn, 1999; Fulkerson et al., 2004; Post, 2005; Witvrouw et al., 2005; Fagan and Delahunt, 2008), although the true efficacy of many of these treatments has yet to be proven. The lack of certainty about these three factors leaves this question unanswered.

\section{Conclusion}

To the best of our knowledge, this is the first study to produce population prevalence rates for various patellofemoral disorders across the life course. These data suggest that a significant proportion of adults with knee pain consulting in primary care in the UK are considered by their GPs to have disorders of the PFJ. Routine general practice morbidity recording has clear limitations in its ability to describe the epidemiology of patellofemoral disorders. Population-based studies are needed to fill this evidence gap.

\section{Acknowledgements}

We thank the Keele GP Research Partnership and the Informatics team at the Arthritis Research UK National Primary Care Centre. The 'Consultations in Primary Care Archive' (CiPCA) is funded by the North Staffordshire Primary Care Research Consortium and the National Coordinating Centre for Research Capacity Development (NCCRCD).

\section{References}

Arendt, E. 2005: Anatomy and malalignment of the patellofemoral joint: its relation to patellofemoral arthrosis. Clinical Orthopaedics and Related Research, issue 436, 71-75.

Becker, R., Ropke, M., Krull, A., Musahl, V. and Nebelung, W. 2008: Surgical treatment of isolated patellofemoral osteoarthritis. Clinical Orthopaedics and Related Research 466, 443-49.

Brukner, P., Kahn, K., Crossley, K.M., Cook, J., Cowan, S.M. and McConnell, J. 2007: Anterior knee pain. In Brukner, P. and Kahn, K. editors. Clinical sports medicine, third edition. Sydney: McGraw-Hill. 506-37.

Butcher, J.D., Zukowski, C.W., Brannen, S.J., Fiesler, C., O'Connor, F.G., Farrish, S. and Lillegard, W.A. 1996: Patient profile, referral sources, and consultant utilization in a primary care sports medicine clinic. Journal of Family Practice 43, 556-60.

Crossley, K.M., Marino, G.P., Macilquham, M.D., Schache, A.G. and Hinman, R.S. 2009: Can patellar tape reduce the patellar malalignment and pain associated with patellofemoral osteoarthritis? Arthritis and Rheumatism 61, $1719-25$.

Devereaux, M.D. and Lachmann, S.M. 1984: Patello-femoral arthralgia in athletes attending a sports injury clinic. British Journal of Sports Medicine 18, 18-21.

Donell, S.T. and Glasgow, M.M. 2007: Isolated patellofemoral osteoarthritis. Knee 14, 169-76.

Duncan, R.C., Hay, E.M., Saklatvala, J. and Croft, P.R. 2006: Prevalence of radiographic osteoarthritis-it all depends on your point of view. Rheumatology (Oxford) 45, 757-60.

Duncan, R., Peat, G., Thomas, E., Wood, L., Hay, E. and Croft, P. 2009: Does isolated patellofemoral osteoarthritis matter? Osteoarthritis Cartilage 17, 1151-55.

Englund, M. and Lohmander, L.S. 2005: Patellofemoral osteoarthritis coexistent with tibiofemoral osteoarthritis in a meniscectomy population. Annals of the Rheumatic Diseases 64, 1721-26.

Fagan, V. and Delahunt, E. 2008: Patellofemoral pain syndrome: a review on the associated neuromuscular deficits and current treatment options. British Journal of Sports Medicine 42, 789-95.

Fulkerson, J.P., Buuck, D.A., Dye, S.F., Farr, J. and Post, W.R. 2004: Disorders of the patellofemoral joint, fourth edition. Philadelphia, PA: Lippincott Williams and Wilkins.

Grelsamer, R.P. 2005: Patellar nomenclature: the Tower of Babel revisited. Clinical Orthopaedics and Related Research, issue $436,60-65$. 
Hill, C.L., Gale, D.R., Chaisson, C.E., Skinner, K., Kazis, L., Gale, M.E. and Felson, D.T. 2003: Periarticular lesions detected on magnetic resonance imaging: prevalence in knees with and without symptoms. Arthritis and Rheumatism 48, 2836-44.

Hinman, R.S. and Crossley, K.M. 2007: Patellofemoral joint osteoarthritis: an important subgroup of knee osteoarthritis. Rheumatology 46, 1057-62.

Hunter, D.J., March, L. and Sambrook, P. 2003: The association of cartilage volume with knee pain. Osteoarthritis and Cartilage 11, 725-29.

Jordan, K., Porcheret, M. and Croft, P. 2004: Quality of morbidity coding in general practice computerized medical records: a systematic review. Family Practice 21, 396-412.

Jordan, K., Porcheret, M., Kadam, U.T. and Croft, P. 2006: The use of general practice consultation databases in rheumatology research. Rheumatology (Oxford) 45, 126-28.

Jordan, K., Clarke, A.M., Symmons, D.P.M., Fleming, D., Porcheret, M., Kadam, U.T. and Croft, P. 2007: Measuring disease prevalence: a comparison of musculoskeletal disease using four general practice consultation databases. British Journal of General Practice 57, 7-14.

Jordan, K.P., Kadam, U.T., Hayward, R., Porcheret, M., Young, C. and Croft, P. 2010: Annual consultation prevalence of regional musculoskeletal problems in primary care. BMC Musculoskeletal Disorders 11, 144.

Juhn, M.S. 1999: Patellofemoral pain syndrome: a review and guidelines for treatment. American Family Physician 60, 2012-22.

Kalichman, L., Zhang, Y., Niu, J., Goggins, J., Gale, D., Felson, D.T. and Hunter, D. 2007: The association between patellar alignment and patellofemoral joint osteoarthritis features-an MRI study. Rheumatology (Oxford) 46, 1303-308.

Kornaat, P.R., Bloem, J.L., Ceulemans, R.Y.T., Riyazi, N., Rosendaal, F.R., Nelissen, R.G., Carter, W.O., Hellio Le Graverand, M.-P. and Kloppenburg, M. 2006: Osteoarthritis of the knee: association between clinical features and MR imaging findings. Radiology 239, 811-17.

LaBella, C. 2004: Patellofemoral pain syndrome: evaluation and treatment. Primary Care 31, 977-1003.

Lanyon, P., O'Reilly, S., Jones, A. and Doherty, M. 1998: Radiographic assessment of symptomatic knee osteoarthritis in the community: definitions and normal joint space. Annals of the Rheumatic Diseases 57, 595-601.

Luhmann, S.J., Schoenecker, P.L., Dobbs, M.B. and Eric, G.J. 2008: Adolescent patellofemoral pain: implicating the medial patellofemoral ligament as the main pain generator. Journal of Children's Orthopaedics 2, 269-77.

Macnicol, M.F. 1995: The problem knee, second edition. Oxford: Butterworth-Heinemann.

McAlindon, T.E. 1999: Regional musculoskeletal pain. The knee. Baillière's best practice \& research. Clinical rheumatology 13, 329-44.
McCormick, A., Fleming, D. and Charlton, J. 1995: Morbidity statistics from general practice. Fourth National Study 1991-1992. London: HMSO.

Neuman, P., Kostogiannis, I., Friden, T., Roos, H., Dahlberg, L.E. and Englund, M. 2009: Patellofemoral osteoarthritis 15 years after anterior cruciate ligament injury-a prospective cohort study. Osteoarthritis and Cartilage 17, 284-90.

Peat, G., McCarney, R. and Croft, P. 2001: Knee pain and osteoarthritis in older adults: a review of community burden and current use of primary health care. Annals of the Rheumatic Diseases 60, 91-97.

Pihlajamaki, H.K., Kuikka, P.I., Leppanen, V.V., Kiuru, M.J. and Mattila, V.M. 2010: Reliability of clinical findings and magnetic resonance imaging for the diagnosis of chondromalacia patellae. The Journal of Bone and Joint Surgery. American Volume 92, 927-34.

Porcheret, M., Hughes, R., Evans, D., Jordan, K., Whitehurst, T., Ogden, H. and Croft, P. 2004: Data quality of general practice electronic health records: the impact of a program of assessments, feedback, and training. Journal of the American Medical Informatics Association 11, 78-86.

Post, W.R. 2005: Anterior knee pain: diagnosis and treatment. Journal of the American Academy of Orthopaedic Surgeons 13, 534-43.

Royal College of General Practitioners' Birmingham Research Unit. 2007: Weekly Returns Service Annual Prevalence Report. London: Royal College of General Practitioners.

Sandow, M.J. and Goodfellow, J.W. 1985: The natural history of anterior knee pain in adolescents. The Journal of bone and joint surgery. British volume 67, 36-38.

Stanitski, C.L. 1993: Anterior knee pain syndromes in the adolescent. The Journal of Bone and Joint Surgery. American Volume 75, 1407-416.

Stathopulu, E. and Baildam, E. 2003: Anterior knee pain: a long-term follow-up. Rheumatology (Oxford) 42, 380-82.

Szebenyi, B., Hollander, A.P., Dieppe, P., Quilty, B., Duddy, J., Clarke, S. and Kirwan, J.R. 2006: Associations between pain, function, and radiographic features in osteoarthritis of the knee. Arthritis and Rheumatism 54, 230-35.

The National Collaborating Centre for Chronic Conditions. 2008: Osteoarthritis. National Clinical Guideline for Care and management in adults. London: Royal College of Physicians..

Urwin, M., Symmons, D., Allison, T., Brammah, T., Busby, H., Roxby, M., Simmons, A. and Williams, G. 1998: Estimating the burden of musculoskeletal disorders in the community: the comparative prevalence of symptoms at different anatomical sites, and the relation to social deprivation. Annals of the Rheumatic Diseases 57, 649-55.

Utting, M.R., Davies, G. and Newman, J.H. 2005: Is anterior knee pain a predisposing factor to patellofemoral osteoarthritis? Knee 12, 362-65. 
van der Waal, J.M., Bot, S.D., Terwee, C.B., van der Windt, D.A., Schellevis, F.G., Bouter, L.M. and Dekker, J. 2006: The incidences of and consultation rate for lower extremity complaints in general practice. Annals of the Rheumatic Diseases 65, 809-15.

van Middelkoop, M., van, L.R., Berger, M.Y., Koes, B.W. and Bierma-Zeinstra, S.M. 2008: Knee complaints seen in general practice: active sport participants versus non-sport participants. BMC Musculoskeletal Disorders 9, 36.
Witvrouw, E., Werner, S., Mikkelsen, C., Van Tiggelen, D., Vanden Berghe, L. and Cerulli, G. 2005: Clinical classification of patellofemoral pain syndrome: guidelines for nonoperative treatment. Knee Surgery, Sports Traumatology, Arthroscopy: Official Journal of the ESSKA 13, 122-30.

Zhang, H., Kong, X.Q., Cheng, C. and Liang, M.H. 2003: A correlative study between the prevalence of chondromalacia patellae and sports injury in 4068 students. Chinese Journal of Traumatology 6, 370-74. 